\title{
Genotyping of the causative Leptospira in symptomatic dogs in Thailand
}

\author{
Weena Paungpin ${ }^{1}$, Somjit Chaiwattanarungruengpaisan ${ }^{1}$, Chalisa Mongkolphan ${ }^{1}$, \\ Witthawat Wiriyarat ${ }^{1,2}$, Metawee Thongdee ${ }^{1 *}$
${ }^{1}$ The Monitoring and Surveillance Center for Zoonotic Diseases in Wildlife and Exotic Animals, Faculty of Veterinary Science, Mahidol University, Salaya, Nakhon Pathom 73170, Thailand
${ }^{2}$ Department of Preclinic and Applied Animal Science, Faculty of Veterinary Science, Mahidol University, Salaya, Nakhon Pathom 73170, Thailand

\begin{abstract}
We investigated the genotypes of Leptospira spp. detected in symptomatic dogs in Thailand. During April to December 2012, 6 out of 41 client-owned dogs were diagnosed with leptospirosis based on polymerase chain reaction tests. All of the infected dogs showed clinical symptoms related to leptospirosis. Direct genotyping of the causative agent of the canine leptospirosis was conducted from the archival DNA samples extracted from urine or blood of those 6 infected dogs. Sequencing of the partial 16S rRNA and lipL32 genes from all samples identified Leptospira (L.) interrogans as the infecting species. Multilocus sequence typing tests were successful for 2 out of 6 samples. The sequence type (ST) was identified as ST50 for both samples where the profile corresponded to L. interrogans species and Bataviae serogroup. The presence of this genotype of Leptospira has never been reported in Thailand. Thus, our findings showed the existence of ST50 L. interrogans serogroup Bataviae and the ability to cause leptospirosis in dogs in Thailand.
\end{abstract}

Keywords: Leptospira, genotype, leptospirosis, dog, MLST

*Corresponding author

Metawee Thongdee

The Monitoring and Surveillance Center for Zoonotic Diseases in Wildlife and Exotic Animals, Faculty of Veterinary Science, Mahidol University, Salaya, Nakhon Pathom 73170, Thailand

Tel: +66-2-441-5236

Fax: +66-2-441-5236

E-mail: metawee.tho@mahidol.ac.th

ORCID:

Weena Paungpin

https://orcid.org/0000-0002-3909-2884

Somjit Chaiwattanarungruengpaisan

https://orcid.org/0000-0002-0705-9131

Chalisa Mongkolphan

https://orcid.org/0000-0002-9925-5083

Witthawat Wiriyarat

https://orcid.org/0000-0002-0058-0477

Metawee Thongdee

https://orcid.org/0000-0001-9082-0325

Conflict of Interest

There is no conflict of interest.

Received: July 8, 2019

Revised: October 25, 2019

Accepted: December 2, 2019

\section{Introduction}

Leptospirosis is a zoonotic disease affecting humans and animals worldwide [1]. The disease is caused by the infection of spirochete bacteria belonging to the genus Leptospira. Once infected, the bacteria may be maintained in nature by mammalian reservoir hosts. Asymptomatic reservoir hosts can harbor leptospires in their proximal renal tubules and excrete the bacteria through the urine into the environment [2]. Dogs can be infected with Leptospira and served as either maintenance or incidental hosts depending on the infecting serovars [3]. Canine leptospirosis is associated with a variety of clinical manifestations ranging from mild to multi-systemic and fulminant, which can potentially lead to the death of the infected animal [3]. The clinical features of canine leptospirosis include renal, liver and musculoskeletal diseases, bleeding disorders, respiratory diseases and uveitis $[4,5]$.

The major species causing leptospirosis in dogs are Leptospira (L.) interrogans and Leptospira kirschneri $[3,6]$. In the past, the most common serogroups affecting dog populations were Canicola and Icterohaemorrhagiae. However, the widespread usage of the bivalent Canicola and Icterohaemorrhagiae vaccines together with the increased exposure to wild or domestic animal reservoir hosts possibly contributed to the emergence of the new infecting serogroups in the dog populations [3]. Nowadays, several serogroups have been recognized as canine pathogens. These include serogroups such as Grippotyphosa, Pomona, Australis and Autumnalis [3,7]. In addition, the infecting serogroups or serovars may vary across different geographical locations [3]. For instance, serogroup Hebdomadis appears to be a predominant infecting serogroup causing high mortality among infected dogs in Japan [8] while serogroup Icterohaemorrhagiae is a major causative agent of canine leptospirosis in Brazil [9].

In Thailand, the information regarding the infecting serogroups in dogs was very limited. Therefore, this study aimed to investigate the genotypes of the 
causative Leptospira in symptomatic dogs found in Thailand. The molecular characterization based on 16S rRNA and lipL32 genes as well as multilocus sequence typing (MLST) were conducted using the archival DNA samples from infected dogs that were previously diagnosed with leptospirosis.

\section{Materials and Methods}

\section{Ethics statement}

This study was approved by the Ethics and Animal Care and Use Committee of the Faculty of Veterinary Science, Mahidol University (Permit Number: MUVS-2019-02-04).

\section{Archived DNA samples}

DNA samples used in the study were from a collection of diagnostic accessions between April and December 2012 submitted to the Monitoring and Surveillance Center for Zoonotic Diseases in Wildlife and Exotic animals, Faculty of Veterinary Science, Mahidol University. The DNA samples were extracted from the urine or blood of the client-owned dogs presented to the Faculty's animal hospital. Dogs with a clinical suspicion of leptospirosis may exhibit high serum blood urea nitrogen (BUN) and creatinine (CR) levels with unknown origin ( $>60 \mathrm{mg} / \mathrm{dL}$ and $1.4 \mathrm{mg} / \mathrm{dL}$ respectively) and showed two or more clinical manifestations suggestive of leptospirosis including jaundice, vomiting, hemorrhagic disorders, fever, prostration, hyporexia/anorexia [8,9]. The clinical signs of dogs were recorded at the time of presentation and blood samples were subjected to biochemistry and hematological parameters estimation. These parameters include BUN and CR serum concentrations, alkaline phosphatase (ALP) and alanine aminotransferase activity (ALT), hematocrit (Ht) and white blood cell (WBC) count. The samples obtained from dogs with clinical diagnosis of leptospirosis based on a combination of clinical signs and a positive urine or blood polymerase chain reaction (PCR) tests were used in this study.

\section{DNA extraction and PCR assays}

Each urine sample was initially centrifuged at $2,000 \times \mathrm{g}$ for $5 \mathrm{~min}$ to discard cell debris and supernatant was subsequently centrifuged at $14,000 \times \mathrm{g}$ for $20 \mathrm{~min}$ to collect pellet. DNA extraction from the urine pellets was performed with QIAamp Viral RNA Mini Kit (QIAGEN, Germany) following the manufacturer's instruction. DNA extraction from EDTA anticoagulated blood samples was undertaken with DNeasy blood and tissue kit (QIAGEN) according to the manufacturer's recommendation.

PCR assays targeting 16S rRNA and lipL32 genes were used to detect leptospiral DNA in urine or EDTA anticoagulated blood of dogs involved in this study. An amplification of the highly conserved $16 \mathrm{~S}$ rRNA gene, which is used for species identification of Leptospira, was carried out by using genus specific primers $\mathrm{F}$ (5' GGCGGCGCGTCTTAAACATG 3') and R (5' TTCCCCCCATTGAGCAAGATT 3') [10].
A total volume of $25 \mu \mathrm{L}$ PCR reaction contained $1 \times \mathrm{MgCl}_{2}$ free PCR buffer, $2.5 \mathrm{mM}$ of $\mathrm{MgCl}_{2}, 200 \mu \mathrm{M}$ of dNTP, 1.25 unit of Taq DNA polymerase (iNtRON Biotechnology Inc, Korea), $0.5 \mu \mathrm{M}$ of each primer, and $5 \mu \mathrm{L}$ of DNA extracted from clinical samples. The PCR conditions were as follows: one cycle of initial denaturation at $94^{\circ} \mathrm{C}$ for $3 \mathrm{~min}, 30$ cycles of denaturation at $94^{\circ} \mathrm{C}$ for $1 \mathrm{~min}$, annealing at $63^{\circ} \mathrm{C}$ for 1.5 $\mathrm{min}$, and elongation at $72^{\circ} \mathrm{C}$ for $2 \mathrm{~min}$; followed by one cycle of final extension at $72^{\circ} \mathrm{C}$ for $10 \mathrm{~min}$. DNA of $L$. interrogans serovars Copenhageni was included as a positive control and nuclease-free water was included as a negative control in each PCR run. The amplified PCR products of $331 \mathrm{bp}$ were analyzed by using $1.5 \%$ agarose gel electrophoresis.

An amplification of the lipL32 gene, which is present only in the pathogenic Leptospira spp. was conducted using primers LipL32-45F (5' AAGCATTACCGCTTGTG GTG 3') and LipL32-286R (5' GAACTCCCATTTCAGCGATT 3') [11]. PCR reactions were prepared in a volume of $25 \mu \mathrm{L}$ containing all components as mentioned above. The PCR conditions were as follows: one cycle of initial denaturation at $94^{\circ} \mathrm{C}$ for $2 \mathrm{~min}$; 30 cycles of denaturation at $94^{\circ} \mathrm{C}$ for $1 \mathrm{~min}$, annealing at $57^{\circ} \mathrm{C}$ for $45 \mathrm{sec}$, and elongation at $72^{\circ} \mathrm{C}$ for 45 sec; followed by one cycle of final extension at $72^{\circ} \mathrm{C}$ for 10 min. Positive and negative controls of each run were included. The amplified products of $242 \mathrm{bp}$ in size were analyzed by using $1.5 \%$ agarose gel electrophoresis.

\section{S rRNA and lipL32 gene sequencing and phyloge- netic analysis}

The amplified PCR products were cloned into pGEM-T Easy Vector (Promega, USA) based on TA cloning and transformed into Escherichia coli competent DH5a cells. The plasmid DNA containing desired insertion was isolated from a positive clone and the target DNA was sequenced at the SolGent (Korea). The trimmed nucleotide sequences of the amplicons were deposited into the GenBank database. Nucleotide sequence analysis was conducted using the National Center for Biotechnology Information (NCBI) BLAST. A phylogenetic tree was constructed based on partial nucleotide sequences of 16S rRNA (331 bp) and lipL32 gene (242 bp) by using the Neighbor-Joining method with the Kimura 2-parameter model in the MEGA program version 7.0.

\section{Multilocus sequence typing}

Genotyping of pathogenic Leptospira was performed by MLST according to the previously described method [12]. Briefly, seven housekeeping loci consisting of $g \operatorname{lm} U$, pntA, sucA, tpiA, pfkB, mreA, caiB were amplified individually from the DNA samples using a primer set for each target gene. The PCR reaction mixtures were prepared as described above. Amplification conditions were as follows: one cycle of $95^{\circ} \mathrm{C}$ for $2 \mathrm{~min}, 30$ cycles of $95^{\circ} \mathrm{C}$ for $10 \mathrm{sec}, 46^{\circ} \mathrm{C}$ for 15 $\mathrm{sec}$, and $72^{\circ} \mathrm{C}$ for $30 \mathrm{sec}$, followed by a final extension of $72^{\circ} \mathrm{C}$ for $7 \mathrm{~min}$. The PCR products with expected size were purified from agarose gel by using GenepHlowTM Gel/PCR 

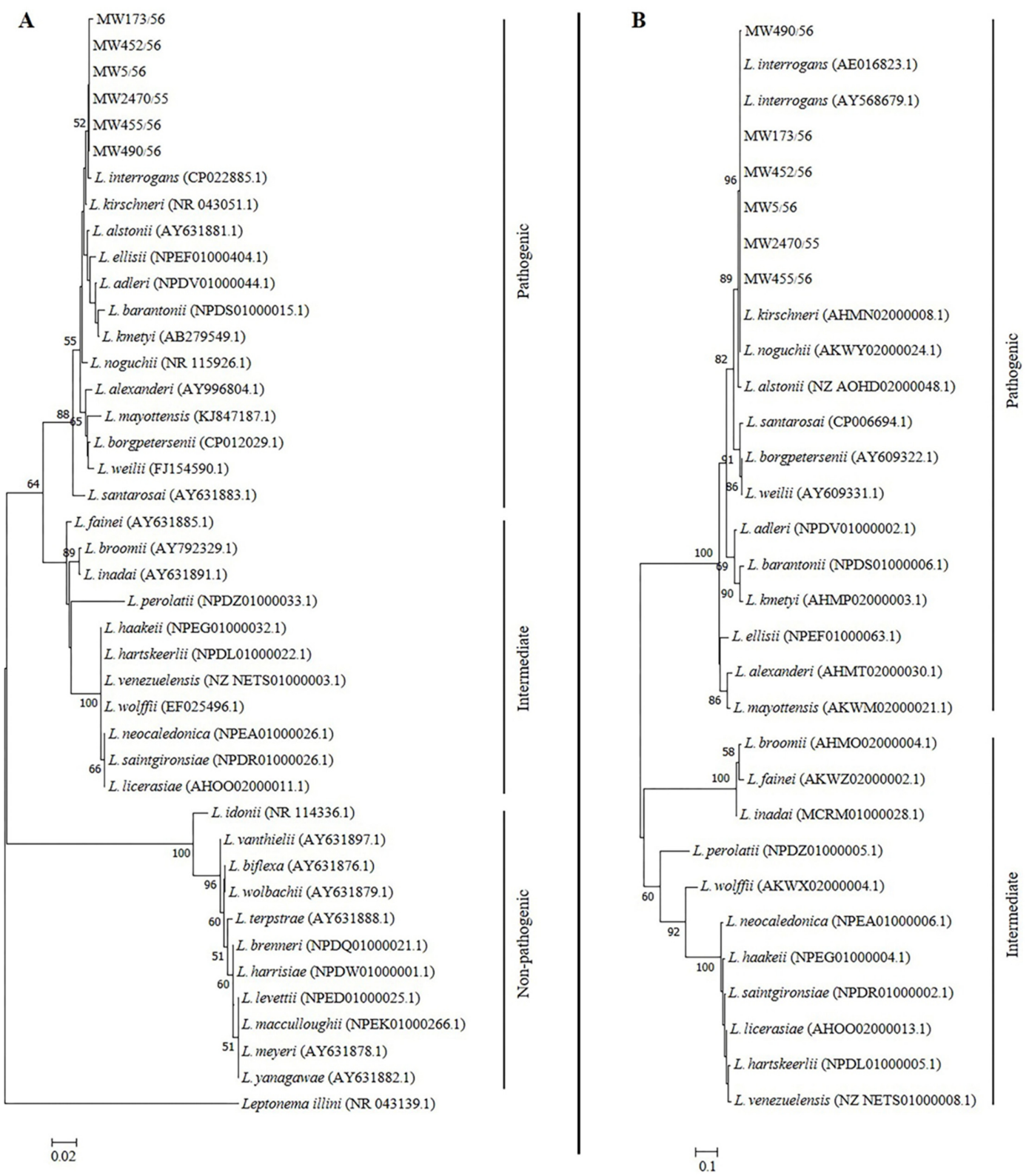

Fig. 1. Neighbor joining phylogenetic trees of partial nucleotide sequences of (A) 16S rRNA and (B) lipL32 of Leptospira amplified from urine or blood samples of symptomatic dogs. A bootstrap of 1000 replicates was performed and values above $50 \%$ are shown in the nodes. Leptonema illini was used as the outgroup for the 16S rRNA tree. Reference species and their GenBank accession numbers are included.

Kit (Geneaid, Taiwan) according to the manufacturer's instruction. The purified PCR products were subsequently sequenced. The trimmed nucleotide sequences with the correct length for each locus were submitted into the Leptospira MLST database (https://pubmlst.org/leptospira/) to identify the allele numbers. Allelic profiles; in the order $g \operatorname{lm} U-p n t A-$ sucA-tpiA-pfkB-mreA-caiB, were used to determine the sequence type (ST) for each sample. The relationship among STs listed on the Leptospira MLST database was analyzed using eBURST algorithm (http://eburst.mlst.net/).

\section{Results}

During April to December, 2012, a total of 41 clinical samples (urine $=40$; blood $=1$ ) from client-owned dogs were submitted for laboratory diagnosis of leptospirosis. Of those, 6 clinical samples (urine $=5$; blood $=1$ ) were found to be PCR positive for leptospiral DNA. All of the PCR positive samples were derived from sick dogs with suspected leptospirosis. The remaining clinical samples, which were free from leptospiral DNA, were originated from 2 groups; sick dogs with suspected leptospirosis $(n=12)$ and healthy dogs 
4 Weena Paungpin, Somjit Chaiwattanarungruengpaisan, Chalisa Mongkolphan, Witthawat Wiriyarat, Metawee Thongdee

$(n=23)$.

Among the group of infected dogs with confirmed leptospirosis, the ages of the animals ranged from 4 months to 8.8 years (median 21 months) and 67\% were female. Two animals were unvaccinated while the rest of the animals had uncertain vaccination history. The clinical symptoms of the infected dogs presented at the first hospital visit included jaundice (100\%), anorexia (83\%), depression (67\%), vomiting $(67 \%)$, diarrhea (33\%) and organomegaly (33\%). Laboratory analyses revealed average BUN and CR levels of 201 $\mathrm{mg} / \mathrm{dL}(\mathrm{SD}, 142.71)$ and $8.18 \mathrm{mg} / \mathrm{dL}$ (SD, 5.73). The average ALT and ALP serum activities were 96.75 IU/L (SD, 76.11) and 619.33 IU/L (SD, 380.40). Lastly, the average Ht count was $30.88 \%(\mathrm{SD}, 6.43)$ and $\mathrm{WBC} / \mu \mathrm{L}$ was $40,700(\mathrm{SD}$, 18,333.03). The clinical outcome of 4 infected dogs were death while the clinical outcome of the other two dogs could not be determined due to the lack of follow-up care. The infected dogs were from the west districts of Bangkok and the neighboring Nakhon Pathom province.

Six leptospiral DNA-positive samples obtained from the infected dogs were used for further molecular characterizations. All of the 6 samples (MW2740/55, MW5/56, MW173/ 56, MW452/56, MW455/56 and MW490/56) were tested positive based on PCRs using both genus-specific primers targeting 16S rRNA gene and pathogenic-specific primers targeting lipL32 genes (Table 1). The 16S rRNA and lipL32 amplicons from those samples were sequenced and the resulting nucleotide sequences were deposited into the GenBank database under accession number MK558418-MK558423 and MK558360-MK558365 respectively. In all cases, the

Table 1. Molecular characterization of Leptospira based on 16S rRNA, lipL32 and MLST

\begin{tabular}{|c|c|c|c|c|c|c|}
\hline \multicolumn{2}{|c|}{ Canine host } & \multirow[b]{2}{*}{ DNA source } & \multirow[b]{2}{*}{$16 \mathrm{~S}$ rRNA } & \multirow[b]{2}{*}{ lipL32 } & \multirow[b]{2}{*}{ Species } & \multirow[b]{2}{*}{ MLST } \\
\hline ID & $\begin{array}{c}\text { Clinical } \\
\text { outcome }\end{array}$ & & & & & \\
\hline MW2470/55 & Death & Urine & + & + & L. interrogans & NA \\
\hline MW5/56 & Death & Urine & + & + & L. interrogans & NA \\
\hline MW173/56 & Unknown & Urine & + & + & L. interrogans & ST50 L. interrogans Bataviae \\
\hline MW452/56 & Unknown & Urine & + & + & L. interrogans & ST50 L. interrogans Bataviae \\
\hline MW455/56 & Death & Urine & + & + & L. interrogans & NA \\
\hline MW490/56 & Death & Blood & + & + & L. interrogans & NA \\
\hline
\end{tabular}

Leptospira interrogans, L. interrogans; NA, not applicable.

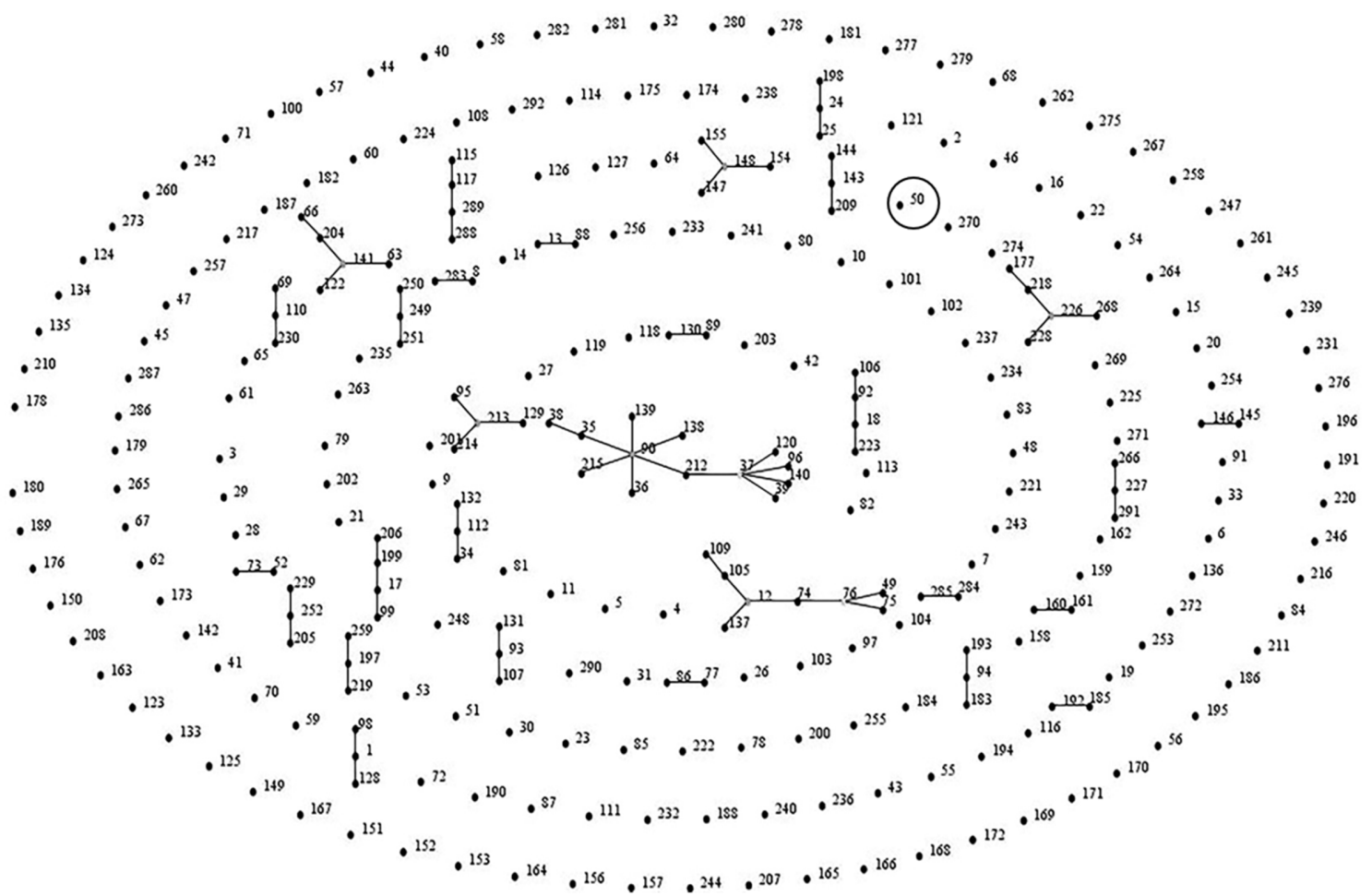

Fig. 2. eBURST analysis of the causative Leptospira interrogans detected in symptomatic dogs in Thailand. The sequence type of the detectable Leptospira is indicated by a black circle. 
16S rRNA and lipL32 amplicon sequences exhibited greater than $99 \%$ similarities with sequences of the pathogenic Leptospira found in the GenBank database. The phylogenetic tree analyses based on the partial nucleotide sequences of 16S rRNA (Fig. 1A) and lipL32 (Fig. 1B) demonstrated that the amplicon sequences from all samples were clustered with the pathogenic leptospiral clade that is related to L. interrogans.

Genotyping by using MLST was successful for two samples, which are MW173/56 and MW452/56. The amplification of seven house-keeping genes for the other 4 samples were unsuccessful due to insufficient DNA. The identical allelic profiles generated from the seven loci was obtained for both samples. The profile in the order $g \operatorname{lmU}$-pntA-sucAtpiA-pfkB-mreA-caiB: 6-8-2-2-9-7-5 was identified as ST50 (Table 1), which corresponds to L. interrogans species and Bataviae serogroup. The eBURST analysis of the entire 292 STs listed on the Leptospira MLST database (Accessed July 2019) demonstrated that ST50 was a singleton on the eBURST diagram (Fig. 2) indicating that the strain had no relationship with the other Leptospira spp.

\section{Discussion}

This study uncovered the genotype of the causative Leptospira detected from canine leptospirosis cases presented in 2012. Based on molecular characterizations, ST50 L. interrogans serogroup Bataviae was found responsible for the clinical illness and, possibly, death of the infected dogs. The obvious clinical sign exhibited by all of the six infected dogs was jaundice. The mechanism to induce jaundice by pathogenic Leptospira is considered to arise from the disruption of the intercellular junction of the host hepatocytes through Leptospira invasion resulting in leakage of bile [13]. Although serogroup Bataviae is unrecognized and scarcely evidenced to cause disease through natural infection in dogs, the experimental infection by L. interrogans serovars Bataviae in dogs showed severe illness variably associated with renal, hepatic and hemorrhagic manifestations [14]. However, the severity of the disease in naturally occurring canine leptospirosis may be dependent on other factors such as the age and immune status of the hosts, the virulence of the infecting serovars/ serogroups and geographical location [3].

In this study, the infecting species was identified from all samples by using 16S rRNA and lipL32 gene sequencing. The genotype of the infecting species was successfully determined for 2 out of 6 samples by using MLST analysis. We lacked the genotypes information for the Leptospira that caused the death of the other 4 infected dogs due to the insufficient DNA amount in the samples, which was a limitation of our study. Hence, our findings could not completely confirm the relationship between the genotype of the causative Leptospira and the virulence relevant to the identified genotypes. Nevertheless, our findings indicated the existence of ST50 L. interrogans serogroup Bataviae and the ability to cause disease in dogs found in Thailand. The source of infec- tion should be further investigated to identify a natural reservoir for this canine pathogen. An earlier study reported that rodents in urban Bangkok (central Thailand) were found to carry Leptospira serovar Bataviae in their kidneys as shown by direct immunofluorescent assay. However the genotype of the bacteria remains unknown [15]. Although the presence of ST50 L. interrogans has never been reported in Thailand, this Leptospira genotype was found to be a predominant clone circulating in rodents and other small mammal populations in several areas of Malaysia, particularly in the residential areas where suspected cases of human leptospirosis had been frequently reported [16,17]. In recent studies, L. interrogans serogroup Bataviae has been isolated from kidneys of Rattus norvegicus captured in Hanoi [18] and Hai Phong [19], Vietnam. The multilocus variable-number of tandem repeat analysis (MLVA) showed that the Bataviae serogroup isolates were identical to that of the strains causing severe leptospirosis in the 2009 outbreak in the Philippines [18]. On the other hand, the molecular epidemiological survey of leptospirosis in Laos and Cambodia demonstrated that rodents captured in the urban and rural areas of both countries were reservoir for pathogenic Leptospira especially L. interrogans [20,21]. The MLVA typing profiles of these bacteria were identical to the reference strains belonging to the Canicola and Pyrogenes serogroups [20].

To date, the information regarding infecting serogroups or serovars in the local dog population in Thailand is still limited. The available information is primarily from the antibody detection using MAT assay. The data obtained from this method can provide the broad idea of the common serogroup presented in a population [22]. Previous seroprevalence studies reported that Leptospira serogroup Bataviae was the most predominant serogroup in stray dogs in Bangkok and the central region of Thailand accounting for $20.3 \%$ of all the seropositive samples [23]. Similarly, the same serogroup was also found in client-owned dogs in Chiang Mai province (located in the northern region of Thailand) accounting for 5.2\% of seropositive samples [24]. On the other hand, Leptospira serogroup Canicola appeared to be the main serogroup circulating in dogs found in Mahasarakram province (located in the northeastern region of Thailand) accounting for $10.9 \%$ of all seropositive samples [25]. Our genotyping data is in agreement with the earlier serosurveys where Leptospira serogroup Bataviae was shown to be the local serogroup that was causing leptospirosis in dogs in Thailand. It is a fact that both molecular genotyping and serotyping methods have the benefit of facilitating the epidemiological studies and surveillance as well as supporting outbreak investigation of canine leptospirosis as shown in the previous studies that were carried out in Thailand [26,27].

Our study provided genotyping data of Leptospira detected from symptomatic dogs with confirmed leptospiral infection. Although the study is limited by the small sample size, the genotype information of infecting Leptospira strain described here will be important and useful for the establishment of 
6 Weena Paungpin, Somjit Chaiwattanarungruengpaisan, Chalisa Mongkolphan, Witthawat Wiriyarat, Metawee Thongdee

appropriate controls and preventive measures for canine leptospirosis in Thailand. In this case, the currently available vaccines may not fully protect dogs in Thailand since they do not provide cross protection against serovars not included in the vaccines [28]. In addition, increasing the awareness for the dog owners and veterinarians about the disease even in the vaccinated dogs should be considered. Future studies should investigate the genetic diversity of pathogenic Leptospira in the relevant natural reservoirs to elucidate the source of infection and disease transmission in dogs.

\section{Acknowledgements}

This work was financially supported by the Faculty of Veterinary Science, Mahidol University, Salaya, Nakhon Pathom, Thailand.

\section{References}

1. Levett PN. Leptospirosis. Clin Microbiol Rev 2001;14:296326.

2. Bharti AR, Nally JE, Ricaldi JN, Matthias MA, Diaz MM, Lovett MA, Levett PN, Gilman RH, Willig MR, Gotuzzo E, Vinetz JM; Peru-United States Leptospirosis Consortium. Leptospirosis: a zoonotic disease of global importance. Lancet Infect Dis 2003;3:757-771.

3. Sykes JE, Hartmann K, Lunn KF, Moore GE, Stoddard RA, Goldstein RE. 2010 ACVIM small animal consensus statement on leptospirosis: diagnosis, epidemiology, treatment, and prevention. J Vet Intern Med 2011;25:1-13.

4. André-Fontaine G. Canine leptospirosis--do we have a problem? Vet Microbiol 2006;117:19-24.

5. Lunn KF. Clinical manifestations of leptospirosis in dogs. 26th Annual Forum of the American College of Veterinary Internal Medicine Conference 2008 (ACVIM 2008). Available from: https://www.vin.com/apputil/content/defaultadv1.aspx?id= 3865654\&pid=11262. Accessed July 5, 2019.

6. Schuller S, Francey T, Hartmann K, Hugonnard M, Kohn B, Nally JE, Sykes J. European consensus statement on leptospirosis in dogs and cats. J Small Anim Pract 2015;56: 159-179.

7. Klaasen HL, van der Veen M, Molkenboer MJ, Sutton D. A novel tetravalent Leptospira bacterin protects against infection and shedding following challenge in dogs. Vet Rec 2013;172: 181 .

8. Koizumi N, Muto MM, Akachi S, Okano S, Yamamoto S, Horikawa K, Harada S, Funatsumaru S, Ohnishi M. Molecular and serological investigation of Leptospira and leptospirosis in dogs in Japan. J Med Microbiol 2013;62:630-636.

9. Miotto BA, Tozzi BF, Penteado MS, Guilloux AG, Moreno LZ, Heinemann MB, Moreno AM, Lilenbaum W, Hagiwara MK. Diagnosis of acute canine leptospirosis using multiple laboratory tests and characterization of the isolated strains. BMC Vet Res 2018;14:222.

10. Mérien F, Amouriaux P, Perolat P, Baranton G, Saint Girons I. Polymerase chain reaction for detection of Leptospira spp. in clinical samples. J Clin Microbiol 1992;30:2219-2224.

11. Stoddard RA, Gee JE, Wilkins PP, McCaustland K, Hoffmaster AR. Detection of pathogenic Leptospira spp. through TaqMan polymerase chain reaction targeting the LipL32 gene. Diagn Microbiol Infect Dis 2009;64:247-255.

12. Boonsilp S, Thaipadungpanit J, Amornchai $\mathrm{P}$, Wuthiekanun $\mathrm{V}$, Bailey MS, Holden MT, Zhang C, Jiang X, Koizumi N, Taylor K, Galloway R, Hoffmaster AR, Craig S, Smythe LD, Hartskeerl RA, Day NP, Chantratita N, Feil EJ, Aanensen DM, Spratt BG, Peacock SJ. A single multilocus sequence typing (MLST) scheme for seven pathogenic Leptospira species. PLoS Negl Trop Dis 2013;7:e1954.

13. Miyahara S, Saito M, Kanemaru T, Villanueva SY, Gloriani NG, Yoshida S. Destruction of the hepatocyte junction by intercellular invasion of Leptospira causes jaundice in a hamster model of Weil's disease. Int J Exp Pathol 2014;95: 271-281.

14. Keenan KP, Alexander AD, Mongomery CA Jr. Pathogenesis of experimental Leptospira interrogans, serovar bataviae, infection in the dog: microbiological, clinical, hematologic, and biochemical studies. Am J Vet Res 1978;39:449-454.

15. Doungchawee G, Phulsuksombat D, Naigowit P, Khoaprasert Y, Sangjun N, Kongtim S, Smythe L. Survey of leptospirosis of small mammals in Thailand. Southeast Asian J Trop Med Public Health 2005;36:1516-1522.

16. Benacer D, Mohd Zain SN, Ahmed AA, Mohd Khalid MK, Hartskeerl RA, Thong KL. Predominance of the ST143 and ST50 Leptospira clones in the urban rat populations of Peninsular Malaysia. J Med Microbiol 2016;65:574-577.

17. Azhari NN, Ramli SN, Joseph N, Philip N, Mustapha NF, Ishak SN, Mohd-Taib FS, Md Nor S, Yusof MA, Mohd Sah SA, Mohd Desa MN, Bashiru G, Zeppelini CG, Costa F, Sekawi Z, Neela VK. Molecular characterization of pathogenic Leptospira sp. in small mammals captured from the human leptospirosis suspected areas of Selangor state, Malaysia. Acta Trop 2018;188:68-77.

18. Koizumi N, Miura K, Sanai Y, Takemura T, Ung TT, Le TT, Hirayama K, Hasebe F, Nguyen HL, Hoang PV, Nguyen CN, Khong TM, Le MT, Hoang HT, Ohnishi M. Molecular epidemiology of Leptospira interrogans in Rattus norvegicus in Hanoi, Vietnam. Acta Trop 2019;194:204-208.

19. Koizumi N, Izumiya $\mathrm{H}$, Mu JJ, Arent Z, Okano S, Nakajima C, Suzuki Y, Mizutani Muto M, Tanikawa T, Taylor KR, Komatsu N, Yoshimatsu K, Thi Thu Ha H, Ohnishi M. Multiple-locus variable-number tandem repeat analysis of Leptospira interrogans and Leptospira borgpetersenii isolated from small feral and wild mammals in East Asia. Infect Genet Evol 2015;36:434-440.

20. Cosson JF, Picardeau M, Mielcarek M, Tatard C, Chaval Y, Suputtamongkol Y, Buchy P, Jittapalapong S, Herbreteau V, Morand S. Epidemiology of leptospira transmitted by rodents in southeast Asia. PLoS Negl Trop Dis 2014;8:e2902.

21. Kudo Y, Vansith K, Rin E, Uchida K, Kodama S, Fukui T, Masuda S, Masuzawa T. Molecular epidemiological survey of Leptospira infection of wild rodents in the urban settlement of Cambodia. Vector Borne Zoonotic Dis 2018;18:144-150.

22. Levett PN. Usefulness of serologic analysis as a predictor of the infecting serovar in patients with severe leptospirosis. Clin Infect Dis 2003;36:447-452.

23. Jittapalapong S, Sittisan P, Sakpuaram T, Kabeya H, Maruyama $\mathrm{S}$, Inpankaew T. Coinfection of Leptospira spp and Toxoplasma gondii among stray dogs in Bangkok, Thailand. Southeast Asian J Trop Med Public Health 2009;40:247-252. 
24. Meeyam T, Tablerk P, Petchanok B, Pichpol D, Padungtod P. Seroprevalence and risk factors associated with leptospirosis in dogs. Southeast Asian J Trop Med Public Health 2006;37: 148-153.

25. Pumipuntu N, Suwannarong K. Seroprevalence of Leptospira Spp. in Cattle and Dogs in Mahasarakham Province, Thailand. J Health Res 2016;30:223-226.

26. Kurilung A, Chanchaithong P, Lugsomya K, Niyomtham W, Wuthiekanun V, Prapasarakul N. Molecular detection and isolation of pathogenic Leptospira from asymptomatic humans, domestic animals and water sources in Nan province, a rural area of Thailand. Res Vet Sci 2017;115:146-154.

27. Thongdee $M$, Chaiwattanarungruengpaisan $S$, Lekcharoen $P$, Yimchoho N, Buathong R, Wiriyarat W. A novel genotype of Leptospira interrogans recovered from leptospirosis outbreak samples from southern Thailand. Jpn J Infect Dis 2019;72: 343-346.

28. Eric Klaasen HL, Adler B. Recent advances in canine leptospirosis: focus on vaccine development. Vet Med (Auckl) 2015;6:245-260. 